\title{
Expression Alteration of Disrupted in Schizophrenia 1 (DISC1) Gene, a Potential Peripheral Marker for Schizophrenia and Paranoid Personality Disorder
}

Jalal Rostampour ${ }^{1}$, Arvin Haghighatfard ${ }^{2,3 *}$, Masoume Ghasemzadeh Qazvini ${ }^{4}$, Talie Karimi ${ }^{5}$, Elham Rastegarimoghaddam ${ }^{6}$, Atieh Alizadenik ${ }^{7}$ and Zahrasadat Hosseini ${ }^{8}$

${ }^{1}$ Department of Cell and Molecular Biology, School of Biology, College of Science, University of Tehran, Tehran, Iran

${ }^{2}$ Department of Biology, Tehran North branch, Islamic Azad University, Tehran, Iran

${ }^{3}$ Young Researchers and Elites Clubs, Tehran North Branch, Islamic Azad University, Tehran, Iran

${ }^{4}$ Department of Biology, Faculty of Sciences, University of Guilan, Rasht, Iran

${ }^{5}$ Department of Biology, Ashkezar Branch, Islamic Azad University, Yazd, Iran

${ }^{6}$ Department of Microbiology, Damghan Branch, Islamic Azad University, Damghan, Iran

${ }^{7}$ Department of Biology, Damghan Branch, Islamic Azad University, Damghan, Iran

${ }^{8}$ Department of Biological Science, Kharazami University, Karaj, Iran

\begin{abstract}
Introduction: Schizophrenia (SCZ) is a major psychiatric disorder with unclear etiology or biological diagnosis. Paranoid Personality Disorder (PPD) is a type A personality disorder characterized by paranoia and generalized mistrust. Disrupted in schizophrenia 1 (DISC1) is a gene located on human chromosome 1 that is involved in neurodevelopment of brain. Variations and translocations in this gene were found associated with schizophrenia and other psychiatric disorders. Present study aimed to evaluate the expression alteration of DISC1 gene in peripheral blood of SCZ and PPD patients and its correlation with clinical features.
\end{abstract}

Material and methods: Study was included 300 SCZ, 300 PPD and 300 non-psychiatric individuals. Total blood was collected and expression level of DISC1 evaluated by using quantitative Real time PCR SYBR green. Lymphocyte DISC1 protein levels in all subjects were examined. Also, to assess psychiatric symptoms severity, Positive and Negative Syndrome Scale (PANSS) was obtained from SCZ and PPD patients. For analysis of executive functions abilities, Wisconsin Card Sorting Test (WCST) had been conducted from all subjects.

Results: Findings showed significant DISC1 gene down expression in SCZ and PPD patients vs. non-psychiatrics. DISC1 protein levels were significantly decreased in SCZ and PPD vs. non-psychiatrics. Also in SCZ patients, general and negative symptoms score were associated with down regulation of DISC1 mRNA level. Executive functions deficiency had detected in SCZ and PPD and correlations were found between decrease in WSCT correct response and down expression of DISC1 in SCZ and PPD patients.

Discussion and conclusion: Results presented DISC1 as potential peripheral marker for schizophrenia as well as paranoid personality disorder. Correlation between DISC1 mRNA level reduction and severity of general and negative symptoms in one side and executive functions abnormalities in the other side may support the neurodevelopment hypothesis about pathophysiology of schizophrenia and related personality disorder especially paranoid personality disorder.

Keywords: Schizophrenia; Paranoid personality disorder; DISC1; Gene expression

\section{Introduction}

Schizophrenia (SCZ) is a major neuropsychiatric disorder with estimated $1 \%$ prevalence worldwide. Etiology of schizophrenia is unknown, and diagnosis is depending only on descriptive symptomatic and psychiatric interviews. On the other hand, SCZ shows great symptomatic heterogeneity in presence and severity of positive, negative and cognitive symptoms, which lead to so much complexities in diagnosis and treatment of patients [1,2]. Positive symptoms are included hallucinations, delusions and paranoia, negative symptoms are included affective flattening, lack of motivation, poor speech and social withdrawal and cognitive impairments comprise attention deficits disrupted memory functions and sever impairments in executive functions [3]. Clinical symptoms of schizophrenia mostly onset, during adolescence or early adulthood, that may support the evidences about neurodevelopmental disturbance role in pathophysiology of SCZ. Schizophrenia and related complex psychiatric disorders may represent the end point of several different pathogenic pathways. Post mortem studies of schizophrenia could strength neurodevelopmental model due to detected pre-existing morphological abnormalities in the brains of schizophrenic patients at the onset of the condition. In addition, psychiatric reports show that schizophrenics demonstrate several behavioral abnormalities and executive function deficiencies in childhood years before the onset of symptoms [4].
Paranoid personality disorder (PPD) is a type A personality disorder characterized by paranoia and a pervasive, longstanding suspiciousness and generalized mistrust of others. Prevalence of Paranoid personality disorder was estimated about $0.5 \%$ to $2.5 \%$ in general population in different countries. No clear etiology or molecular mechanism was suggested for PPD but the heritability of this disorder is high [5]. First molecular genetics study on Paranoid personality disorder were detected several shared genetic biomarkers between SCZ and PPD in mitochondrial complex I [6].

Disrupted In Schizophrenia 1 (DISC1), originally discovered as the putative gene disrupted on chromosome 1 by a balanced translocation

*Corresponding author: Arvin Haghighatfard, Department of Biology, Tehran North Branch, Islamic Azad University, Tehran, Iran, Tel: +98 212240 4843; E mail: arvinland@yahoo.com

Received August 22 2017; Accepted November 22, 2017; Published November 28, 2017

Citation: Rostampour J, Haghighatfard A, Qazvini MG, Karimi T, Rastegarimoghaddam E, et al. (2017) Expression Alteration of Disrupted in Schizophrenia 1 (DISC1) Gene, a Potential Peripheral Marker for Schizophrenia and Paranoid Personality Disorder. Hereditary Genet 6: 188. doi:10.4172/21611041.1000188

Copyright: (c) 2017 Rostampour J, et al. This is an open-access article distributed under the terms of the Creative Commons Attribution License, which permits unrestricted use, distribution, and reproduction in any medium, provided the original author and source are credited. 
Citation: Rostampour J, Haghighatfard A, Qazvini MG, Karimi T, Rastegarimoghaddam E, et al. (2017) Expression Alteration of Disrupted in Schizophrenia 1 (DISC1) Gene, a Potential Peripheral Marker for Schizophrenia and Paranoid Personality Disorder. Hereditary Genet 6: 188. doi:10.4172/2161-1041.1000188

Page 2 of 4

$\mathrm{t}(1 ; 11)(\mathrm{q} 42.1 ; \mathrm{q} 14.3)$ is one of the hundreds of genes that have been considered as possible risk factors for schizophrenia. DISC1 was co segregates with major psychiatric illness in a great Scottish pedigree, Finnish cohort and Taiwanese families [4]. The DISC1 protein in interaction with number of proteins regulates several cellular functions and molecular pathways, including neuronal migration (LIS1, NDE1, NDEL1, Dixdc1), axonal bundling and elongation (FEZ1), neural progenitor proliferation (GSK3 $\beta$ ), cell cycle regulation (PCM1), neuronal signaling (Girdin, GSK3 $\beta, \mathrm{PDE} 4)$, and signal transduction (Kal7, TNIK, AKAP9) [7]. Mouse models indicate the role of DISC1 protein in neurodevelopment of several brain regions, such as olfactory bulb, cortex, hippocampus, hypothalamus, cerebellum and brain stem and morphological development of adult-born granule neurons $[4,8]$. In human peripheral blood few studies were conducted and down expression of DISC1 genes and some of the DISC1-interactome genes such as NDEL1 has been reported in schizophrenic subjects compared to non-psychiatric controls [7]. Cytoarchitectual alteration in the hippocampus is a neuropathological marker for schizophrenia. Neuronal size reduction and alterations in presynaptic and dendritic markers suggest abnormalities in the hippocampal neural circuitry in schizophrenia as well as some other psychiatric disorders. It is note that the expression of DISC1 was abundant in hippocampal neurons at the developing stage and neuron pruning, may support the potential involvement of DISC1 in the formation of the hippocampal neural circuits [9].

Present study aimed to assess the expression level of DISC1 mRNA in the adult schizophrenic patients and paranoid personality disorder patients in compare with non-psychiatric patients. In addition, correlation of DISC1 mRNA level regulation with severity of psychiatric symptoms and executive functions capabilities had been evaluated.

\section{Material and Methods}

\section{Subjects selections, ethical issues}

The study was included 300 unrelated SCZ patients (180 male, 120 female) and 300 unrelated PPD patients (196 male, 104 female), diagnosed by two independent senior psychiatrists based on extended clinical interview and patients' DSM-V chart review. All of the patients had started treatment, including antipsychotic medications, in the 18-24-month period before sampling and were considered as chronic patients. Patients were selected from psychiatric hospitals and outpatient clinics of IR Iran. Patients with co morbid psychiatric disorders and schizoaffective or schizotypal patients were excluded. Estimated daily dose average of antipsychotics was calculated by using Chlorpromazine (CPZ) equivalents; and estimated lifetime $\mathrm{CPZ}$ equivalents considering of duration of illness. The non-psychiatric group was included 300 nonpsychiatric subjects ( 175 male, 125 female) with matched demographic parameters (such as sex, age, race, socioeconomic situation, familial situation and education) with both patient groups and with no history of any psychological problems, no current or history of severe medical conditions, neurological disorder, history of head trauma with loss of consciousness, no drug abuse and alcohol or nicotine dependence. Subjects were given an explanation on the aim of the study. Finally, before the beginning of study written informed consent was provided according to the Declaration of Helsinki. The study was approved by central ethical committee of Islamic Azad University in Tehran.

\section{Sampling and gene expression evaluation}

Blood samples $(5 \mathrm{ml})$ were collected from the cubital vein without tourniquet using PAXgene blood RNA tubes (Cat No762165) between 10.00 and $11.00 \mathrm{~h}$ and RNA extraction started immediately after sampling. Total RNA was extracted from peripheral blood samples according to the column based standard protocols of the RNA Purification kit (GeneJETTM RNA Purification Kit\#K0732, Fermentas, Latvia). Total RNA was treated with DNase to prevent the contaminating genomic DNA using DNase Treatment and Removal Reagents (DNase I, RNase-free (\#EN0521) Fermentas, Latvia), according to the manufacturer's protocol. The quality and integrity of extracted RNA was examined by gel electrophoresis and UV spectroscopy, and sampling repeated for subjects with lowquality RNA in the first sample. Then cDNA was synthesized with a Transcription First Strand cDNA Synthesis Kit (RevertAid Premium First Strand cDNA Synthesis Kit \#K1652, Fermentas, Latvia) according to the manufacturer's protocol. Primers for reference and interest genes designed by 'oligo7' software and blasted on the NCBI website and checked in In-Silico PCR of UCSC Genome Browser website. PCR and agarose gel electrophoresis were used to verify the predicted size of PCR amplicons of genes. Standard curves for each gene were prepared using serial dilutions (1:4) of pooled cDNA from total RNA extracted from blood samples of 10 non-psychiatric subjects. In each experiment, the R2 value of the standard curve was more than 0.99 , and no-template control assays resulted in no detectable signal. Quantitative real-time PCR (qPCR) was performed using SYBR green (Thermo Scientific Maxima SYBR Green/ROX qPCR Master Mix (2X) \#K0221, Fermentas, Latvia). A triplicate method was performed for quantitative real-time PCR using a 7900HT Fast Real-Time PCR System with a Fast 96-Well Block Module (Applied Biosystems, Foster City, CA, USA). PCR data were obtained by Sequence Detector Software (SDS version 2.3 Rev C Patch, Applied Biosystems) and quantified by the standard curve method. SDS software plotted the real-time fluorescence intensity and selected the threshold within the linear phase of the amplicon profile and drew a standard curve of the cycle at threshold versus extracted RNA quantity. Samples were measured in one plate for one target gene and their $\mathrm{Cq}$ values were in the linear range of the standard curve. In qPCR tests, outliers or sample failures were repeated for each gene. The ratio was calculated using the pfafle formula. For normalization of qPCR experiment HNRNPD gene were used as endogenous reference gene. Primers of DISC1 and HNRNPD genes were presented in Table 1.

\section{Protein level assessment}

Lymphocyte pellets were thawed on ice and immediately lyzedin VRL buffer: $50 \mathrm{mM}$ HEPES ( $\mathrm{pH} 7.5$ ), $250 \mathrm{mM}$ sucrose, $5 \mathrm{mM}$ $\mathrm{MgCl}_{2}, 100 \mathrm{mM} \mathrm{KAc}, 2 \mathrm{mM}$ PMSF (all Sigma-Aldrich, Germany), $2 \mathrm{x}$ Pro-tease Inhibitor (Roche, Germany) supplemented with $1 \%$ TritonX-100, $1 \mathrm{mM}$ PMSF (Sigma-Aldrich, Germany) and $40 \mathrm{U} / \mathrm{mL}$ DNaseI(Roche, Germany). After $40 \mathrm{~min}$ on ice, for DNA digestion the lysate was incubated for $40 \mathrm{~min}$ at $37^{\circ} \mathrm{C}$. Then, the protein content of the lysate was detected by using the DC Protein Assay Kit (Bio-Rad, Germany) and for each sample, 30 microgram of total protein were loaded onto $10 \%$ SDS-PAGE gels. After blotting, the membranes were directly treated with $100 \mathrm{mM} \mathrm{KOH}$ for $5 \mathrm{~min}$ at room temperature and subsequently blocked with $5 \%$ milk in PBS with $0.05 \%$ Tween-20 (PBST). DISC1 immunoreactivity was tested with the human DISC1 specific mAB14F2. Analysis was done blind with regard to diagnosis. Protein level analysis were conducted based on previous studies [10]

\begin{tabular}{|c|c|}
\hline Genes primer & Primer sequence \\
\hline$H N R N P D$ forward primer & 5'AGGGGCATCCCAGGATAGT3' \\
\hline HNRNPD reverse primer & 5'ATGGTGACGGCTAAAGGTGG3' \\
\hline DISC1 forward primer & 5' CCCTTTCGAGGTGAGGGAA3' \\
\hline DISC1 reverse primer & 5' ACGGATCCAAACCCCAAGC3' \\
\hline
\end{tabular}

Table 1: Primers of DISC1 and HNRNPD genes, used for quantitative real time PCR. 
Citation: Rostampour J, Haghighatfard A, Qazvini MG, Karimi T, Rastegarimoghaddam E, et al. (2017) Expression Alteration of Disrupted in Schizophrenia 1 (DISC1) Gene, a Potential Peripheral Marker for Schizophrenia and Paranoid Personality Disorder. Hereditary Genet 6: 188. doi:10.4172/2161-1041.1000188

Page 3 of 4

\section{Psychiatric symptoms examination}

Symptoms severity was measured with the Positive and Negative Syndrome Scale (PANSS). PANSS is a well-known psychiatric test with 30 -item semi-structured interview that assess three major symptom categories associated with schizophrenia: positive, negative and general symptoms. Seven positive symptoms, seven negative symptoms and sixteen general symptoms have been measured in PANSS rating scale [11]. PANSS test were obtained from SCZ and PPD patients by senior psychiatrist.

\section{Executive functions assessment}

The Wisconsin Card Sorting Test (WCST) is a neuropsychological test that widely uses to assess the following 'frontal' lobe functions and study of executive functions including memory, attention and planning. A number of stimulus cards are presented to the participant. In present study the subjects were asked to sort 64 cards to match either color (red, blue, yellow, or green), form (crosses, circles, triangles, or stars), or number of figures (one, two, three, four). During the task, the sorting rule changed discreetly from color to form or number of figures by examiner without the participants being informed. But the participant had been informed that the last card is correctly matched due to the current sorting rule or not. The participants had to shift sets accordingly and sort cards following the new sorting rule. Wisconsin Card Sorting Test was conducted based on previous studies for all subjects by senior psychologist $[3,12]$.

\section{Statistical analysis}

Normal distribution for continuous variables was checked via the Kolmogorov-Smirnov exam. Multiple group comparisons were calculated by one-way ANOVA followed by independent Student's t-test. Pearson correlation test was conducted to indicate the relationship between clinical and genetic variables. Statistical significance was set at $\mathrm{P}<0.05$ and Bonferroni correction test was used for multiple comparisons corrections. To control of any potential confounds, body mass index (BMI), medication classes, RNA quality and concentration, cDNA synthesis quality, plates/runs of qPCR and primer efficiency were added as covariates and persistence of the significant difference in main effect between groups was assessed by ANCOVA. Statistical analysis conducted by using SPSS version 23 .

\section{Results}

Demographic and clinical data (mean \pm SD) presented for each group in Table 2.

\section{DISC1 gene expression analysis}

Significant down expression of mRNA levels of DISC1 were

\begin{tabular}{|c|c|c|c|}
\hline Variables & SCZ & PPD & $\begin{array}{c}\text { Non- } \\
\text { psychiatric }\end{array}$ \\
\hline Age & $32 \pm 4$ & $34 \pm 3$ & $31 \pm 6$ \\
\hline PANSS total & $74.42 \pm 14.31$ & $51.65 \pm 10.27$ & - \\
\hline General symptoms & $31.32 \pm 5.82$ & $26.16 \pm 3.24$ & - \\
\hline Positive score & $27.38 \pm 4.56$ & $24.71 \pm 7.26$ & - \\
\hline Negative score & $14.76 \pm 5.44$ & $7.14 \pm 6.12$ & - \\
\hline $\begin{array}{c}\text { WSCT correct } \\
\text { responses }\end{array}$ & $56.24 \pm 18.5$ & $61.25 \pm 13.04$ & $79.36 \pm 6.39$ \\
\hline $\begin{array}{c}\text { WSCT completed } \\
\text { groups }\end{array}$ & $2.2 \pm 1.2$ & $2.18 \pm 1.9$ & $3.92 \pm 1.6$ \\
\hline
\end{tabular}

SCZ: Schizophrenia; PPD: Paranoid Personality Disorder; PANSS: Positive and Negative Syndrome Scale; WSCT: Wisconsin Card Sorting Test

Table 2: Demographic and clinical characteristics of SCZs, PPDs and nonpsychiatrics.

\begin{tabular}{|c|c|c|c|}
\hline $\begin{array}{c}\text { Ratio and statistical } \\
\text { analysis }\end{array}$ & $\begin{array}{c}\text { SCZ vs. non- } \\
\text { psychiatric }\end{array}$ & $\begin{array}{c}\text { PPD vs. non- } \\
\text { psychiatric }\end{array}$ & SCZ vs. PPD \\
\hline Ratio & $0.40 \pm 0.16$ & $0.53 \pm 0.13$ & $0.89 \pm 0.22$ \\
\hline P value & 0.001 & 0.002 & 0.28 \\
\hline F value & 19.38 & 16.77 & 4.22 \\
\hline
\end{tabular}

SCZ: Schizophrenia; PPD: Paranoid Personality Disorder

Table 3: DISC1 gene expression ratio and statistical results for each group comparisons.

detected in peripheral blood of schizophrenic patients $(\mathrm{P}=0.001)$ and patients with paranoid personality disorder $(\mathrm{P}=0.002)$ in compare with non-psychiatric subjects. No significant difference was found in regulation of DISC1 mRNA levels in schizophrenic patients versus paranoid personality disorder subjects. $\mathrm{P}$ values and ratio for each comparison have presented in Table 3.

\section{DISC1 protein level evaluation}

Immunoblotting by monoclonal antibody $14 \mathrm{~F} 2$ which is almost completely specific for human DISC1 [9] and densitometric examination blind of $100 \mathrm{kDa}$ DISC1 immunoreactive band revealed a significant reduction of DISC1 protein in schizophrenia (SCZ) and paranoid personality patients (PPD) patients in compare with nonpsychiatric subjects. Protein level examination has confirmed gene expression results.

\section{Psychiatric results analysis}

Scores of positive, negative and general symptoms for SCZ and PPD groups were presented in Table 2. Significant correlations were found between general psychiatric symptoms score ( $p$ value $=0.002$ and $R$ value $=-0.66$ ) and negative symptoms score ( $p$ value $=0.001$ and $R$ value $=-0.59$ ) of PANSS with mRNA level of DISC1 in SCZ patients. No significant correlations were found between PANSS scores and down regulation DISC1 in PPD patients.

\section{Results of executive functions examination}

Wisconsin Card Sorting Test results showed significant deficiency in executive functions in SCZ ( $\mathrm{p}$ value $=0.001$ ) and PPD ( $\mathrm{p}$ value $=0.001)$ patients in compare with non-psychiatric individuals. Significant negative correlation had been detected between decrease in WSCT correct responses and down regulation of DISC1 mRNA level in SCZ ( $\mathrm{p}$ value $=0.004$ and $\mathrm{R}$ value $=0.74$ ) and $P P D$ ( $\mathrm{p}$ value $=0.009$ and $R$ value $=0.56)$ patients.

\section{Discussion}

Several independent genetic association and mouse model studies indicate DISC1 as a potential risk gene for schizophrenia. Recent studies suggest a possible involvement of DISC1 in neurodevelopment. DISC1 interacts with number of neuronal proteins like NUDEL and FEZ1, play a significant role in neurite outgrowth of PC12 cells, is important in cytoskeletal elements and is related to regulation of neuronal migration in association with NUDEL-Lis1 complex $[4,13]$. While schizophrenia has a complex pattern of functional and neuropathological abnormalities including reduced brain mass and increased ventricular volume, deficiency in DISC1 functions could be presented as a strong etiological marker. Expression studies in adult mouse brain showed that DISC1, and may use as marker for molecular events leading to the development and onset of schizophrenia as well as its role in neurodevelopment, and corticogenesis [12].

Findings of present study confirmed the results of limited number of studies that previously assessed the DISC1 mRNA level and protein in peripheral blood of SCZ patients [13]. Uncertainty about similarity 
of gene expression and protein level results in blood and brain is a great obstacle in studies about mental disorders such as neuropsychiatric disorders. By the way previous studies in animal models showed that DISC1 protein level of lymphatic cells and brain are similar and blood can present the protein level in brain [10]. In addition, similar expression changes with SCZs were found in PPD patients that may support the same role of DISC1 in etiology and molecular pathways that lead to onset of PPD and SCZ. While there is a major lack in molecular study in PPD patients, previous study on gene expression of mitochondrial complex I, have been showed a great similarity in altered genes and their direction in SCZ and PPD patients [6]. Also taken together with reports about mitochondrial complex I abnormalities in PPD patients [6] and previous study that revealed DISC1-dependent regulation of mitochondrial dynamics in neuronal dendrites, present findings may suggest a new molecular pathway for PPD onset including DISC1 and mitochondrial complex I subunits.

\section{Conclusion}

Findings raises the possibility that dysfunction of DISC1 may disrupt development of the nervous system, and astrocytes that lead to vulnerability to psychiatric illnesses and dysfunction in executive function and reasonable thinking (that could be the source of paranoid behaviors) respectively. Significant correlations which were detected between clinical features and DISC1 expression level may strength evidences about role of DISC1 in behavioral changes as well as neurodevelopment of brain. DISC1 down expression were correlated with increase in severity of general and negative symptoms, including blunted affects, passive/apathetic social withdrawal, emotional withdrawal, difficulty in abstract thinking and lack of spontaneity and flow of conversation [10]. It seems that all of these symptoms could cause by neuronal dysfunctions that in turn is related to lack of DISC1 protein in different regions of brain. In executive functions assessments DISC1 expression decrease is associated with less correct response in Wisconsin Card Sorting Test in both patients group [14]. WCST is a neuropsychological test of 'set-shifting', which use to assess the following 'frontal' lobe functions and study of executive functions. Interestingly expression alteration of DISC1 in hippocampus and frontal lobe had been reported in schizophrenic animal models [12]. Association between DISC1 expression change and executive function abnormalities was previously reported and present study showed the same pattern in PPD patients. Development of schizophrenia is strongly related to a deregulation in the balance between circulation of neuroexcitatory reproductive hormones to the brain and consequent remodeling of synapses in specific brain areas. Neurons containing reproductive hormones that are relate to etiology of schizophrenia are highly expressed in the hypothalamus, a region that is enriched with DISC1 related neurons. It seems that DISC1 gene could be presented as an important biomarker in molecular mechanisms of schizophrenia as well as paranoid personality disorder.

\section{References}

1. Ben-Shachar D, Karry R (2007) Sp1 expression is disrupted in schizophrenia; A possible mechanism for the abnormal expression of mitochondrial complex I genes, NDUFV1 and NDUFV2. PLoS ONE 2: e817.

2. Mehler-Wex C, Duvigneau JC, Hartl RT, Ben-Shachar D, Warnke A, et al (2006) Increased mRNA levels of the mitochondrial complex I 75-kDa subunit. Eur Child Adolesc Psychiatry 15: 504-507.

3. Haghighatfard A, Hosseini Z, Mahmoodi A, Mahmoodi A, Qazvini MG, et al (2016) NRG1 types down regulate in methamphetamine and heroin dependent patients. MOJ Addict Med Ther 3: 00030.

4. Schurov IL, Handford EJ, Brandon NJ, Whiting PJ (2004) Expression of disrupted in schizophrenia 1 (DISC1) protein in the adult and developing mouse brain indicates its role in neurodevelopment. Molecular Psych 9: 1100.

5. Waldinger RJ (1997) Psychiatry for medical students. Arlington (VA): American Psychiatric Pub, USA.

6. Haghighatfard A, Andalib S, Amini Faskhodi M, Sadeghi S, Ghaderi AH, et al (2017) Gene expression study of mitochondrial complex I in schizophrenia and paranoid personality disorder. World J Biol Psychiatry 20: 1-14.

7. Rampino A, Walker RM, Torrance HS, Anderson SM, Fazio L, et al. (2014) Expression of DISC1-interactome members correlates with cognitive phenotypes related to schizophrenia. PloS ONE 9: p.e99892.

8. Lee H, Kang E, GoodSmith D, Yoon DY, Song H, et al. (2015) DISC1-mediated dysregulation of adult hippocampal neurogenesis in rats. Front Syst Neurosci 9.

9. Miyoshi K, Honda A, Baba K, Taniguchi M, Oono K, et al. (2003) Disrupted-inschizophrenia 1 , a candidate gene for schizophrenia, participates in neurite outgrowth. Molecular Psych 8: 685-694.

10. Trossbach SV, Fehsel K, Henning U, Winterer G, Luckhaus C, et al. (2014) Peripheral DISC1 protein levels as a trait marker for schizophrenia and modulating effects of nicotine. Behav Brain Res 275: 176-182.

11. Association AP (1994) Diagnostic and statistical manual of mental disorders (DSM), American psychiatric association, Washington, USA. pp. 143-147.

12. Barkley RA (1997) Behavioral inhibition, sustained attention, and executive functions: Constructing a unifying theory of ADHD. Psychological Bull 121: 65.

13. Benes FM, Vincent SL, Todtenkopf M (2001) The density of pyramidal and nonpyramidal neurons in anterior cingulate cortex of schizophrenic and bipolar subjects. Biol Psych 50: 395-406.

14. Norkett R, Modi S, Birsa N, Atkin TA, Ivankovic D, et al. (2016) DISC1dependent regulation of mitochondrial dynamics controls the morphogenesis of complex neuronal dendrites. J Biol Chem 291: 613-629. 